\title{
New ideas about the phase structure of the great circle of blood circulation
}

\begin{abstract}
The article proposes a new phase structure of a large circle of blood circulation for the evaluation of rheovasograms of the upper and lower extremities, which is based not on the geometric characteristics and visual evaluation of rheographic complexes, but on the genesis of their basic elements. We distinguish systole and diastole in the circulatory cycle of the great circle of blood by analogy with the cardiac cycle. Diastole consists of the following phases: the metabolic phase coinciding in time with the diastole of the right atrium, the spread of the pulse wave, rapid inflow and slow inflow; The systole includes the phases: elastic and muscular components of outflow, capillary-venous outflow, coinciding in time with the process of right atrial systole. The proposed phase structure can be used to quantify the biomechanics of a large range of blood circulation through computer rheovasography, calculate a number of parameters of electrical conductivity/tissue resistance and hemodynamics; allows you to connect the biomechanics of a large range of blood circulation with the kinetics of the main arteries and the biomechanics of the heart, to identify causal mechanisms between them, the relationship of central and peripheral hemodynamics, and to evaluate the biomechanics of blood circulation as a whole.
\end{abstract}

Keywords: biomechanics, large circle of blood circulation, rheovasography, phase structure
Volume 2 Issue 3 - 2018

\section{Garanin AA, Ryabov AE}

Samara State Medical University of the Ministry of Health of the Russian Federation, Russia

Correspondence: Garanin AA, Samara State Medical University of the Ministry of Health of the Russian Federation, Samara, Russia, Email: sameagle@yandex.ru

Received: April 27, 2018 | Published: May 17, 2018
Abbreviations: $\mathrm{AH}$, arterial hypertension; DM, diabetes mellitus; MB, metabolic phase; RPV, the phase of the pulse wave propagation; $\mathrm{BP}$, fast flow phase; $\mathrm{ECO}$, phase of the elastic component of the outflow; MKO, muscle component of the outflow; KVO, phase of capillary-venous outflow; SP, atrial systole; DP, diastole of the atria.

\section{Introduction}

The principal development and proposal of the rheographic technique belongs to N. Mann (1937). Subsequently, the technique was developed in the works of AA. Kedrova \& T Yu. Lieberman(1941-1949) etc. Detailed development and implementation of the rheography method into clinical practice is associated with the names of Austrian researchers W. Holzer, K. Polzer and A. Marko. ${ }^{1}$ At the present time, it is visually accepted to distinguish the following elements in the rheographic complex: cataract - the main wave of the rheogram, anacrotic (an upward part of the catacrotic), a vertex, a dicrocus (descending part of the catacroton), an incision, a dicrotic wave. A common technique for interpreting and denoting the elements of the rheogram does not exist. ${ }^{2}$ A number of authors analyze their regularity, the steepness of their anacrots, the character of the top, the shape of the cataract and the dicrut, the depth of the incision, which is highly subjective and, despite the high information content, is of little specificity and can depend on many external and internal factors. ${ }^{3}$ These characteristics do not provide a comprehensive picture of the state of the vascular system, but only of its individual functions: the pulse blood filling of the area under study, indirectly about the magnitude of the shock volume of the blood and the state of the tone of large vessels, the ratio of arterial and venous blood flow, etc. In addition, attempts to interpret rheograms do not take into account the genesis of their basic elements. Moreover, the data on the border of arterial inflow and venous outflow on the rheogram complex are contradictory. Some authors believe that venous outflow begins with the descending part of the anacrotic, others believe that the boundary between the arterial and venous parts of the great circle of blood circulation is the incision. ${ }^{4}$ Therefore, it seemed expedient to develop a phase structure of a large circle of blood circulation, which is based not on the geometric characteristics and visual evaluation of rheographic complexes, but on the interpretation of the genesis of their basic elements and allows to obtain a minimum of indicators for quantitative analysis of various aspects of hemodynamics.

\section{Goal}

The study of the interaction of various departments of a large range of blood circulation during the vascular cycle, clarification of the physiological function of all phases and application of the developed phase structure for the evaluation of biomechanics of the great circulation.

\section{Material and methods}

Registration of rheograms of the upper and lower extremities - rheovasograms was performed on the elon polygraph using a standard, traditional technique using longitudinal rheography. The recording of the curves of the upper and lower rheovasograms was performed in parallel with the sphygmograms of the ulnar and femoral arteries respectively. Bipolar circular electrodes were used to register the rheovasograms. The active electrode (proximal) was placed on the upper limb on the shoulder between the proximal and middle third of it, indifferent (distal) - in the wrist area, on the lower - the active electrode between the proximal and middle 
thirds of the thigh, indifferent - just above the ankles. Calibration of rheovasograms was carried out in amplitude using a calibration pulse, which was obtained by unbalancing the bridge by $0.1 \mathrm{ohms}$. At the registration stage, the automation of the process was carried out with the help of the Reos software package, which allows to adapt the Elon polygraph to a computer, carry out two-channel registration of rheovasograms and tracking curves, select the optimal sampling frequency and create a database on the computer. Employees of the department of propaedeutic therapy of Sam GMU (Ryabov AE, Garanin AA) developed a package of applied programs "Rheograph", which makes it possible to identify a data file containing numerical values of the amplitude of the curves, to conduct its preprocessing (smoothing, inventory), to take into account the frequency sampling and calibration signal level during processing, perform manual setting of the reference points - the boundaries of the phases of the vascular cycle according to the first and second derivative of the rheograms presented on the screen and obtain a copy of the result in the form Stow file.

In the process of analysis of rheograms and their derivatives, we have established that the graph of the second derivative of rheograms recorded on both the upper and lower extremities contains two always identifiable minima, three maxima and two transitions through zero. When repeated recording of rheograms for the same subject, these characteristic points occur in a strictly defined time sequence. And this sequence of alternation of points on the second derivative can be traced on the rheograms of various vascular pools of a large range of blood circulation in different individuals regardless of sex and age: when registering the upper and lower rheovasograms, rheoencephalograms, and rheogeopathograms. We propose a new interpretation of the phase structure of the great circle of blood circulation and the curve of the rheovasogram, based on an analysis of its main elements, and not their geometric characteristics. In this case, in the vascular cycle of the great circle of blood circulation, we select systole and diastole by analogy with the cardiac cycle. The systole of the great circle of circulation consists (Fig. 1) of the phases of the elastic (points 3-4) and muscular (points 4-5) of the outflow component (IVF and MKO) and capillary-venous outflow (points 5-6) (CCE). The diastole of the great circle of blood circulation includes the metabolic phase (MB) (points 6-0), the propagation of the pulse wave (RPV) (points 0-1), fast (points1-2) and slow (points 2-3) inflow (BP and MP). In addition, the diastole (DP) of the right atrium is distinguished on the rheovasogram, which coincides with the phase of the KVO (points 5-6) and the systole (SP) coinciding in time with the part of the MB phase (points 7-0). Their activity does not make a significant contribution to the biomechanics of the great circle of blood circulation, therefore calculation of biomechanical parameters in these phases is not carried out separately. ${ }^{5}$ The systole begins with the IVF phase from the arterial part of the large circle of the circulation. It is caused by a reduction in the elastic structures of the walls of the aorta and its branches and the flow of blood into the microcirculatory bed.( Figure 1).

The next phase - MKO from a large circle of blood circulation is characterized by a contraction of the muscle fibers of the aortic walls and its branches and an increase in the blood filling of the arterioles and capillaries as a result of the prevalence of blood flow over the outflow and the increase in the blood flow velocity in the large circulation. The final systolic phase - CWD is caused by the active suction action of the right ventricle in the fast filling phase and the increase in outflow from the large circle of circulation, with its prevalence above the influx. The onset of this phase coincides with the end of the fast-filling phase of the cardiac cycle, continuing into the slow-filling phase and atrial systoles. The joint venture and DP begin the cycle of the right heart. They coincide in time with the CCE and MB phase respectively, determined mainly by the myocardium of the right heart, as well as the main veins and microcirculatory bed. SP-a non-constant wave on rheovasograms caused by a partial return of blood to the vena cava in systole right atrium, leading to a slowing or cessation of venous outflow and, accordingly, to a drop in electrical resistance in the microcirculatory bed. This is due to the anatomy of the myocardium of the atria - the circular muscle bundles of the deep loop-like layer cover the mouth of the hollow veins and during the systole of the atria interfere with the regurgitation of blood into the vena cava. ${ }^{6}$ However, this block is not sealed, and some of the blood still enters the hollow veins, causing a change in resistance. DP is characterized by increased outflow from the venous section of a large circle of circulation, mainly due to the suction action of the right ventricle.

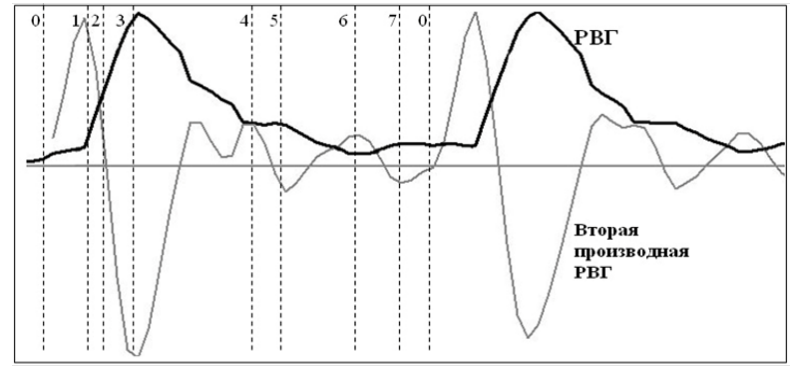

Figure I The phase structure of a large circle of blood circulation (rheogram of the upper limb and its second derivative).

Biomechanics of diastole of a large circle of blood circulation depends on a number of factors: left ventricular systole, aortic and its branches and peripheral resistance of small arteries, arterioles, precapillaries. The diastole of the great circle of blood circulation begins with the MB phase, which has a great physiological meaning. In this phase, the expulsion of blood from the left ventricle and the impulses sent from the sinus node promotes blood flow along the vasa vasorum from the proximal to the distal, ${ }^{7}$ which allows the arteries to prepare for the taking of the shock volume of blood. In addition, it provides trophic tissue to the walls of the main arteries - they are supplied with blood saturated with oxygen and energy products, which makes it possible to synthesize ATP for subsequent active reduction. We noticed that during this period of time, a short increase in electrical conductivity occurs on the rheovasograms, which means that the blood flow through the vessel is not related to the arrival of the main shock volume. A similar phenomenon is not observed either on the rheogram of the pulmonary artery or on a parallel recorded sphygmogram of the main arteries. The next diastolic phase is the phase of the RPV, which characterizes more the mechanical properties of the arterial bed and, to a lesser extent, the left ventricle biomechanics. Phase BP in a large circle of circulation reflects mainly the biomechanics of the left ventricle and the expansion of the aorta and its branches under the impact of the shock volume of blood. The phase of the MP corresponds to the phase of reduced cardiac ejection and reflects the resistance of small vessels of the great circle of blood 
circulation. In each selected phase, we calculated the values of the first and second derivatives of rheovasograms and with their help determined the following parameters of the biomechanics of the great circle of blood circulation:

$$
\begin{aligned}
& \mathrm{t}, \mathrm{c} \text { is the duration of the phase; } \\
& \mathrm{vcp}, \mathrm{Om} / \mathrm{s} \text {-average rate of change of resistance; } \\
& \mathrm{ve}, \mathrm{Om} / \mathrm{s} \text {-the extreme rate of change of resistance; } \\
& \text { asp, Om/ } \mathrm{c}^{2} \text {-average acceleration (force); } \\
& \text { ae, Om/ } \mathrm{c}^{2} \text {-the extreme acceleration (force); }
\end{aligned}
$$$$
\mathrm{Ncp}, \mathrm{Om}^{2} / \mathrm{c}^{3} \text {-average power; }
$$$$
\mathrm{Ne}, \mathrm{Om}^{2} / \mathrm{c}^{3} \text {-extreme power; }
$$$$
\mathrm{W}, \mathrm{Om}^{2} / \mathrm{c}^{2}-\text { work. }
$$

\section{Results and its discussion}

In recent years, there has been a significant increase in interest in the study of morph functional disorders of the arterial bed. The long-held concept that the circulatory system is presented as a closed system of tubes in which the motor that generates the pulse flow is a pump (heart) connected with passive tubes (arteries) is not true. Studies of recent years have shown the inconsistency of this theory, and it is now known that the biomechanics of large arteries is more complex, and the existing model shared the conductive and damping properties of the arteries - the functions inherent only in the aorta. A major contribution to the study of biomechanics of the vascular bed was made by the fundamental works of Russian scientists. Professor M.V. Yanovsky belongs to the theory of the "peripheral heart", which seemed to him as an active systole-diastole of the arteries in a clear and regular combination with the phases of cardiac activity. ${ }^{8} \mathrm{We}$ were convinced of the absolute validity of this theory. We consider it expedient to isolate in the vascular cycle a large circle of blood circulation of two periods -systoles and diastoles.

Analysis of the rheographic curve by means of the proposed phase structure allowed us to reveal the features of blood circulation along a large range of blood circulation in healthy volunteers and individuals with the main risk factors for cardiovascular diseases (Table 1). The results of an analysis of the biomechanics of a large blood circulation of 130 people are presented in four groups: 1 group (40 people) practically healthy persons, 2 group (30 people) - smokers, 3 group (30 people) - persons with arterial hypertension (AH ), 4 group (30 people) - patients with type 2 diabetes mellitus (DM). The systole of the great circle of blood circulation consists of the phases of IVF, $\mathrm{MKO}$ and KVO. The phase of IVF is provided by contraction of the elastic membranes of the aorta and its branches, previously stretched by the shock volume of the left ventricle and coincides with the phases of reduced ejection and isovolumic reduction of intraventricular pressure. There were no significant changes in the duration of this phase in groups 2 and 3 with respect to the norm. In group 4, an increase in the duration of this phase by $10 \%(\mathrm{p}=0.024)$ compared with the control group. In groups 2 and 3, there were no significant changes in the speed and strength of biomechanics of the circulatory system in comparison with the norm and each other. In group 4, a decrease in the mean velocity with respect to the norm by $17 \%$ was found $(\mathrm{p}=0.024)$. Similar dynamics was recorded in the analysis of changes in the average acceleration - it decreased in group 4 compared with the control by $21 \%(\mathrm{p}=0.008)$. In this group there was also a decrease in the average power and work when comparing the results with the norm by $37 \%(\mathrm{p}=0.004)$ and $26 \%(\mathrm{p}=0.022)$, respectively. In addition, in group 3 there was a decrease in work, in comparison with the control group, by $27 \%(\mathrm{p}=0.018)$.

Consequently, in patients with diabetes, there are biomechanical disturbances in the IVF phase, which are primarily related to the functioning of the elastic structures of the arterial part of the large circulation and are decompensated, manifesting a total decrease in all speed and strength indicators. In patients with $\mathrm{AH}$, only a decrease in the vascular wall was detected, and the remaining biomechanical parameters studied did not change significantly. The phase of MKO from a large circle of blood circulation is caused by the contraction of the muscle fibers of the walls of the arteries, corresponds to the phase of rapid filling of the ventricles, as a result of which the blood flow of the microcirculatory bed increases as a result of the prevalence of blood flow over the outflow and the increase in the blood flow velocity in the large circulation. This phase has not revealed any significant changes in its duration and average speed in all groups studied by us. In the $\mathrm{AH}$ group, a decrease in the mean acceleration was found when comparing the results with Groups 1 and 2 by $41 \%(\mathrm{p}=0.009)$ and $33 \%$ $(\mathrm{p}=0.0007)$, respectively. In addition, a decrease in the mean power and work in this group with respect to the norm by $52 \%(\mathrm{p}=0.01)$ and $63 \%(\mathrm{p}=0.014)$, respectively, was recorded.

The final systolic phase - CVC - is due to the continuing outflow of blood from the large circle of circulation to the right atrium, which at this moment is the conductor between the system of hollow veins and the right ventricle, which in its lumen has a minimal pressure towards the end of the rapid filling phase. The sucking action of the right ventricle in the diastole, extending to the venous system of a large circle of blood circulation, ensures the movement of blood in the phase of the CCE. Blood from the capillaries of the microcirculatory bed drains through postcapillaries, venules and veins. The end of this phase coincides with the end of the phase of slow filling of the left ventricle and the onset of the systole of the left atrium. The shortening of this phase in group 2, compared with the norm, by $26 \%(\mathrm{p}=0.031)$ was noted. There was also an increase in the duration of the CCE phase in group 3 when comparing the results with groups 2 and 4 by $30 \%(p=0.034)$ and $15 \%(p=0.022)$, respectively. There was a decrease in the average acceleration in group 3 relative to groups 1 and 2 by $47 \%(\mathrm{p}=0.004)$ and $45 \%(\mathrm{p}=0.007)$, respectively. In group 4 , the mean acceleration decreased by $46 \%(\mathrm{p}=0.001)$ and $44 \%$ $(\mathrm{p}=0.047)$ in relation to the indicated groups, respectively. In group 3 , in addition, the fall in the average power and work, as compared to the norm, was registered by $56 \%(\mathrm{p}=0.008)$ and $60 \%(\mathrm{p}=0.012)$, respectively. Consequently, smokers only found a shortening of the phase of the CCE, which characterizes the dystonia of the distal parts of the arterial part of the great circle of blood under the direct action of nicotine. In patients with diabetes, a significant decrease in strength was found, that is caused, first of all, by the disturbance of microcirculatory bed functioning against the background of this disease. The most pronounced changes were obtained in persons with AH: along with an increase in the duration of the studied phase, a significant decrease in the strength characteristics of the kinetics of a large range of blood circulation was noted, which indicates a violation of the biomechanics of the right heart (its subendo- and subepicardial layers) in the diastole, and dysfunction of the microcirculatory bed. 
Table I Indicators of biomechanics of a large circle of blood circulation according to rheovasography of the upper limb in the studied groups

\begin{tabular}{|c|c|c|c|c|c|c|c|}
\hline $\begin{array}{l}\text { Phases } \\
\text { Groups }\end{array}$ & ME & RPV & BP & MP & ECO & MCO & QUO \\
\hline \multicolumn{8}{|c|}{ The duration of the phases, $t$ (s) } \\
\hline Group 1 & 0,09 & 0,09 & 0,06 & 0,06 & 0,21 & 0,16 & $0,31^{\mathbf{p 1}-2}$ \\
\hline Group 2 & 0,10 & 0,10 & 0,06 & 0,06 & 0,24 & 0,17 & $0,23^{\mathrm{p} 2-3}$ \\
\hline Group 3 & 0,11 & 0,11 & 0,05 & 0,05 & 0,19 & 0,15 & 0,30 \\
\hline Group 4 & 0,10 & 0,10 & 0,06 & 0,06 & $0,23^{\mathbf{p 1 - 4}}$ & 0,15 & $0,26^{\mathbf{p 3 - 4}}$ \\
\hline \multicolumn{8}{|c|}{ The average speed, $\mathrm{v}\left(10^{-5} \mathrm{~Pa} / \mathrm{s}\right)$} \\
\hline Group 1 & 0,59 & 0,80 & 8,08 & 10,67 & 4,95 & 2,06 & 2,13 \\
\hline Group 2 & 0,73 & 0,68 & 7,40 & 10,85 & 4,63 & 1,50 & 2,04 \\
\hline Group 3 & 0,45 & 0,64 & 8,34 & 10,54 & 4,00 & 1,50 & 1,97 \\
\hline Group 4 & 0,44 & 0,91 & 7,74 & 10,55 & $4,13^{p 1-4}$ & 2,13 & 2,31 \\
\hline \multicolumn{8}{|c|}{ The average acceleration, a $\left(10^{-5} \mathrm{~Pa} / \mathrm{s}^{2}\right)$} \\
\hline Group 1 & 6,51 & 65,20 & 138,22 & 107,75 & 110,99 & 51,85 & $31,82^{\mathrm{p} 1-3}$ \\
\hline Group 2 & 9,61 & 62,52 & 139,45 & 106,89 & 95,91 & $45,56^{\mathrm{p2-3}}$ & 30,49 p2-4 \\
\hline Group 3 & 4,86 & 58,50 & 143,20 & 106,17 & 93,60 & $30,75^{\mathrm{p} 1-3}$ & $16,81^{\mathrm{p} 2-3}$ \\
\hline Group 4 & 6,04 & 62,16 & 139,28 & 96,97 p 1-4 & $87,93^{p 1-4}$ & 42,50 & $17,01^{\mathrm{p} 1-4}$ \\
\hline \multicolumn{8}{|c|}{ Average power, $\mathrm{N}\left(10^{-10} \mathrm{~Pa} / \mathrm{s}^{3}\right)$} \\
\hline Group 1 & 2,87 & 62,52 & 1082,19 & 1156,10 & 378,61 & 93,89 & 70,48 \\
\hline Group 2 & 5,68 & 59,55 & 979,23 & 1085,07 & 332,55 & 72,35 & 47,33 \\
\hline Group 3 & 2,17 & 46,52 & 1011,31 & 1084,01 & 304,52 & $45,39^{\mathrm{p} 1-3}$ & $31,29^{\mathrm{p} 1-3}$ \\
\hline Group 4 & $3,18^{\mathrm{p} 2-4}$ & 68,15 & 1066,31 & 1073,23 & $238,86^{\mathrm{p1-4}}$ & 82,56 & 36,81 \\
\hline \multicolumn{8}{|c|}{ Work, W $\left(10^{-10} \mathbf{P a} / \mathbf{s}^{2}\right)$} \\
\hline Group 1 & 0,15 & 4,41 & 41,88 & 46,25 & 54,27 & 12,48 & 7,21 \\
\hline Group 2 & 0,37 & 4,22 & 39,17 & 43,41 & 48,80 & 6,88 & 4,73 \\
\hline Group 3 & 0,16 & 3,85 & 38,66 & 43,56 & $39,82^{\mathrm{p} 1-3}$ & $4,67^{\mathrm{p1}-3}$ & $2,91^{\mathrm{p} 1-3}$ \\
\hline Group 4 & 0,14 & 4,79 & 42,65 & 44,13 & $40,44^{\mathrm{p} 1-4}$ & 7,04 & 3,64 \\
\hline
\end{tabular}

pl-2 significant difference in group 2 with a control group;

$\mathrm{pl}-3$ significant difference in group 3 with the control group

$\mathrm{pl}-4$ significant difference in group 4 with control group;

p2-3 significant difference between 2 and 3 groups;

p2-4 significant difference between 2 and 4 groups;

p3-4 significant difference between 3 and 4 groups.

Diastole of the great circle of blood circulation consists of phases MB, RPV, BP and MP. The diastole of the great circle of blood circulation begins with a phase that coincides in time with the onset of the intraventricular movement of blood, and ends with the end of the phase of the isovoluminal increase in intraventricular pressure. In this phase, only a significantly significant decrease in the average power in group 4 relative to group 2 by $44 \%(p=0.035)$. The phase of the spread of the pulse wave depends, first of all, on the state of the aorta and its branches, and the state of their neuromuscular apparatus and, to a lesser extent, on the function of the left ventricle. In time, this phase coincides with the phase of maximum expulsion 1 . No significant changes in the studied biomechanical parameters with respect to each other in all the studied groups were detected in this phase. The fast inflow phase reflects, first of all, the function of the left ventricle in the phase of maximal ejection of the left ventricle. The resistance of the elastic structures of the aorta and its large branching can be neglected, and the hemodynamic changes due to disorders of the diastole of the circulatory system are compensated to a certain extent in the phase of the RPV. In this phase, there were no significant violations of biomechanical indicators in all study groups.

The slow inflow phase is associated with the further flow of blood into the aortic basin. In time it coincides with the phase of maximal ejection of the left ventricle 2 and reflects the progression of blood to small arteries and arterioles of the great circle of circulation, the total resistance of which considerably exceeds the resistance of the main vessels of the elastic and muscular-elastic types. In groups 2 and 3 , there were no significant changes in the studied parameters of biomechanics in relation to norm. In group 4 there was a decrease in strength, in comparison with the control group, by $10 \%(\mathrm{p}=0.024)$. Thus, in people with risk factors for cardiovascular diseases, biomechanics of the circulatory system in the basin of the brachial artery were disturbed. In patients with diabetes, an increase in arterial stiffness is observed, caused by a violation of the functional state of connective tissue elements of the artery wall. In patients with $\mathrm{AH}$, there was a disturbance in the functioning of smooth mediated myocytes, 
which is associated with an increase in the total peripheral vascular resistance in this condition. In addition, patients with diabetes showed a violation of capillary-venous outflow, which is primarily due to a malfunction of the microcirculatory bed, against the background of the metabolic and structural changes that this disease causes. The most pronounced changes were obtained by us in individuals with hypertension. There was a significant change in the kinetics of a large range of blood circulation, which indicates both a violation of the biomechanics of the right heart (its subendo- and subepicardial layers) in the diastole and of the dysfunction of the microcirculatory bed. In the group of smokers there is only a dystonia of the distal parts of the arterial part of the great circle of blood circulation.

\section{Conclusion}

The use of computer rheography and a new phase structure of rheograms helps to quantify the biomechanics of a large range of blood circulation and allows you to calculate the number of indicators that allows you to evaluate in detail the function of each of its departments.

\section{Acknowledgments}

None.

\section{Conflict of interest}

Authors declare that there is no conflict of interest.

\section{References}

1. Vinogradova TS, Moscow. Instrumental methods of the study of the cardiovascular system. Medicine. 1986. p. 416.

2. Sidorenko GI, Minsk. Instrumental methods in cardiology (Manual). 1994. p. 272.

3. Sawicki NN. Biophysical basis of blood circulation and clinical methods for the study of hemodynamics Moscow. Medicine. 1974. p. 312.

4. Roytberg GE, Strutynsky AV. Laboratory and instrumental diagnosis of diseases of the internal organs. M Bean. 1999. p. 602.

5. Fatenkov VN. New in the biomechanics of the heart, arteries and the pulmonary circulation: monograph. Samara: Samara State tehn Univ; 2009. p. 222.

6. Obrezan AG, Yarovitsky VN. Effect of hemodynamic changes in the vena cava on intracardiac hemodynamics and visceral. St Petersburg. 2002. p. 276.

7. Ivanova VD, Koshev VI, Pirogov VL, et al. Hemodynamic function of the vascular bed in the norm and experiment. Samara. 2002. p. 10.

8. Obrezan AG, Shunkevich TN. The theory of peripheral heart of Professor M. Janowski: classic modern ideas. Bulletin of St Petersburg University. 2008;3(11):14-23. 